03

\title{
Нелинейные эффекты в поле вязких волн, возбужденном пластиной конечных размеров
}

\author{
() В.А. Павлов, А.С. Павловский, Н.Г. Семёнова
}

Санкт-Петербургский государственный университет, 198504 Санкт-Петербург, Россия

e-mail: v.pavlov@spbu.ru, pavlovskii.art@gmail.com, n.g.semenova@spbu.ru

Поступило в Редакцию 26 декабря 2016 г.

В окончательной редакции 9 января 2019 г.

Принято к публикации 27 апреля 2019 г.

Выполнено численное исследование нелинейных нестационарных полей скорости и давления вязкой несжимаемой жидкости вблизи колеблющейся абсолютно жесткой пластины бесконечно малой толщины и ограниченной длины в направлении колебаний. Показано, что ограничение размера пластины вдоль направления ее колебаний приводит к нелинейности задачи даже при малой амплитуде колебаний поверхности пластины. Исследованы поля скорости и давления при больших амплитудах колебаний пластины. Объяснено возникновение потоков вытеснения при колебании пластины бесконечно малой толщины и ограниченной длины. Исследован механизм формирования мгновенных вихревых движений в вязкой жидкости.

Ключевые слова: вязкая волна, источник конечных размеров, число Маха, конвективное ускорение.

DOI: 10.21883/JTF.2019.10.48164.2147

\section{Введение}

Классическое аналитическое рассмотрение поля вязких волн, использующее осциллирующую бесконечную плоскость, проведено в [1]. Однако практическое применение вязких волн подразумевает использование источника конечного размера. На его поверхности существует колебательная тангенциальная относительная компонента скорости жидкости.

Наиболее интересен для практических приложений режим нелинейного волнового движения. Вязковолновой процесс позволяет объяснить интенсификацию тепломассопереноса, улучшение смачивания поверхностей, увеличение адгезии в процессах ультразвуковой металлизации материалов, производимых по технологической схеме „тонкого слоя““ $[2,3]$, увеличение прочности клеевых соединений при воздействии ультразвука $[4,5]$. Дистанционное возбуждение вязких волн находит применение в медицине [6].

Нелинейные вязкие волны предположительно позволят объяснить механизмы возникновения акустических течений, механизмы возникновения продольных аэроупругих колебаний пластин, обтекаемых колеблющимся потоком [7], взаимодействие акустической волны и взвешенных в жидкости частиц [6] и т.п.

В настоящей работе, продолжающей наши исследования вязких волн в задачах разной геометрии $[8,9]$, проведено численное исследование нестационарных нелинейных полей скорости и давления вязкой несжимаемой жидкости при колебаниях в своей плоскости пластины бесконечно малой толщины, конечного размера длины $L$ вдоль направления колебаний, бесконечно протяженной по третьей координате.

\section{Постановка задачи}

Рассмотрим движение вязкой жидкости вблизи поверхности пластины бесконечно малой толщины, совершающей колебания в плоскости $Y Z$ в направлении оси $Y$ и имеющей в этом направлении ограниченный размер $L$. Начало отсчета совместим с геометрическим центром пластины $X=0, Y=0$. Вязкую жидкость будем считать несжимаемой.

Движение вязкой несжимаемой жидкости опишем системой уравнений движения и неразрывности [8]

$$
\left\{\begin{array}{l}
\frac{\partial \mathbf{V}}{\partial t}+(\mathbf{V} \cdot \nabla) \mathbf{V}=-\frac{\nabla P}{\rho_{0}}+v \Delta \mathbf{V} \\
\operatorname{div} \mathbf{V}=0
\end{array}\right.
$$

Здесь $\mathbf{V}(\mathbf{R})$ - вектор скорости жидкости, $\mathbf{R}-$ радиус-вектор с компонентами $X$ и $Y, P$ - давление, $v=\eta / \rho_{0}-$ кинематическая вязкость, $\eta-$ динамическая вязкость, $\rho_{0}=$ const - невозмушенная плотность жидкости.

Колебания пластины (рис. 1) выразим законом изменения скорости жидкости на ее поверхности $\partial S$ (граничное условие прилипания)

$$
\left.\mathbf{V}\right|_{\partial S}=\Theta(t) V_{0} \sin (\omega t) \mathbf{e}_{Y}
$$

Здесь $\Theta(t \geq 0)=1, \Theta(t<0)=0, V_{0}$ - амплитуда колебательной скорости поверхности, $\omega$ - циклическая частота, $\mathbf{e}_{Y}$ - единичный орт в направлении оси $Y$. Граничное условие на бесконечности имеет вид

$$
\left.\mathbf{V}\right|_{\infty}=0 .
$$




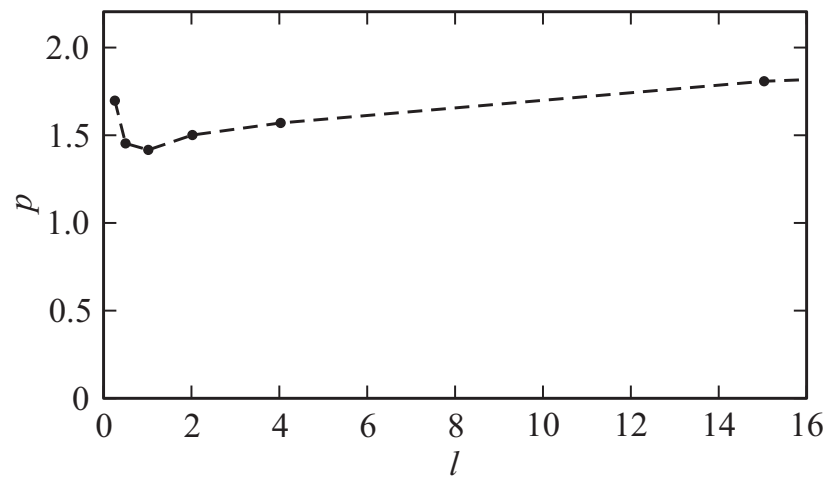

Рис. 1. Зависимость максимальной абсолютной величины поля давления $\max |p|$ от безразмерного параметра $l$ колеблющейся пластины вдоль направления колебаний. Черные кружки - значения параметра $l$, для которого рассчитано значение $\max |p|$. Линия - кусочно-линейная аппроксимация зависимости. Значение параметра $M_{a}=0.5$.

Численно решим задачу (1)-(3) методом конечных элементов (МКЭ), реализованным в Comsol Multiphysics 4.3.

В численной постановке задачи пластина имела нулевую толщину. При решении такой задачи аналитическими или асимптотическими методами исследователи сталкиваются с непреодолимыми математическими трудностями [10], связанными с необходимостью решать полное уравнение (2.2) в окрестности краев пластины [11].

При решении нестационарной нелинейной задачи о распространении вязких волн численно методом конечных элементов тоже возникают трудности. Эти трудности связаны с „некорректностью“ постановки задачи [12]. Исследуемая модель бесконечно тонкой пластины является математической, а не физической. Это приводит к появлению сингулярных точек на краях пластины в численном решении. Здесь величина скорости жидкости терпит разрыв, а величина давления стремится к большой, в теоретическом пределе бесконечной, величине, которая получается при подстановке граничного условия (2) в первое уравнение системы (1).

Прием, известный как сгущение сетки конечных элементов [13], в окрестности края пластины нулевой толщины желаемого результата не дает. Величина давления увеличивается при уменьшении размеров конечных элементов в окрестности края пластины в нескольких последовательных расчетах. Это свидетельствует о том, что в сингулярных точках численная схема решения задачи расходится. Тем не менее указанная трудность не влияет на исследуемые интегральные характеристики полей. Пользуясь МКЭ, можно игнорировать указанную трудность, отступив от краев пластины на расстояние, равное трем размерам конечного элемента [14]. Его безразмерное значение не превышает $\Delta h<0.01$.

Как показывает численное моделирование, в этих точках, лежащих вне окрестности $\Delta h$, величины полей сходятся к конкретным значениям с уменьшением разме- ров конечных элементов в нескольких последовательных расчетах.

Исследования показывают, что без учета функции включения $\Theta$ невозможно корректно поставить задачу, подлежащую решению численными методами. Характерным временем задачи является величина $T=2 \pi / \omega$. Численные решения аналогичных задач $[4,8,9]$ показывают следующую закономерность. После включения колебаний поверхности пластины, в первый интервал времени $t$ от 0 до $T / 2$ поля скорости и давления в вязкой жидкости отличаются от таковых в последующие интервалы времени, равные первому по длительности. В первый интервал времени $t$ движение происходит в изначально покоящейся жидкости. Времена установления полей скорости и давления жидкости составляют не менее трех интервалов времени $T$ на расстоянии порядка характерного размера задачи $\delta=\sqrt{2 v / \omega}$. Отличие величин скорости и давления жидкости в указанной области через $2 T$ после включения колебаний от значений этих величин через $3 T$ после включения колебаний не превосходит $0.01 \%$. В работе [9] оценены времена установления полей скорости и давления в других точках пространства. В настоящей работе представлены результаты, полученные в установившемся режиме.

Поля скорости и давления выражаются через безразмерные функции, найденные в [8]:

$$
\begin{aligned}
& \mathbf{V}=\omega \delta \cdot \mathbf{v}\left(\mathbf{r}, \tau, l \cdot M_{a}\right), \\
& P=\omega \eta \cdot p\left(\mathbf{r}, \tau, l, M_{a}\right) .
\end{aligned}
$$

Здесь

$$
\begin{gathered}
l=\frac{L}{\delta}, \\
M_{a}=\frac{A}{\delta} \frac{V_{0}}{\omega \delta},
\end{gathered}
$$

$\mathbf{r}=\mathbf{R} / \delta-$ безразмерный радиус-вектор с компонентами $x$ и $y, \tau=\omega t-$ безразмерное время, $A-$ размерная амплитуда колебательного смещения пластины.

Масштабом скорости жидкости является величина скорости $c=\omega \delta$ распространения плоской вязкой волны при $l=\infty$. Отношение $V_{0} / c=A / \delta=M_{a}$ (7) является аналогом числа Маха для вязких волн [8].

Существует связь между безразмерными числами $l$ и $M_{a}$ и безразмерными числами Рейнольдса Re и Cтругаля $S_{t}$

$$
\begin{gathered}
R_{e}=2 l M_{a}, \\
S_{t}=\frac{1}{2 \pi} \frac{l}{M_{a}} .
\end{gathered}
$$

Постановка задачи позволяет сравнивать результаты настоящего исследования с результатами предыдущих исследований [8] поля вязких волн, генерируемого цилиндром конечного радиуса и бесконечной длины в направлении своей оси, который поступательно колеблется поперек своей оси. 


\section{Поля давления и скорости жидкости в установившемся режиме колебаний}

В настоящей работе ограничимся описанием установившегося режима полей. Исследование нестационарного процесса включения и развития вязковолновых полей заслуживает отдельного исследования.

В численных экспериментах замечено появление градиентов давления вблизи поверхности колеблющейся пластины ограниченного размера $l$ вдоль направления колебаний и бесконечно малого размера поперек направления колебаний. Появление градиентов давления приводит к возникновению потоков вытеснения и области вытеснения.

Зависимость безразмерной величины давления $p$ от параметра $l$, полученная по результатам численного моделирования, показана на рис. 1.

Введем аналог толщины вытеснения [10] - нестационарную безразмерную величину толщины вытеснения

$$
\tilde{\delta}(\tau)=\int \mathbf{v}\left(\mathbf{r}, \tau, l, M_{a}\right) \mathbf{d r} .
$$

Интегрирование в (10) ведется „поперек“ пограничного слоя на расстояниях по координате $x$ от 0 до 1 .

Поля скорости жидкости, полученные численным моделированием нестационарной нелинейной задачи (1)-(3) при $l \gg 1$ и $M_{a}<1$ отличаются от их значений в линейного приближения на единицы процентов. Известное решение линейной задачи [1] в безразмерном виде имеет вид

$$
\mathbf{v}=M_{a} \exp (-x) \cos (\tau-x) \mathbf{e}_{y} .
$$

Скорость жидкости имеет максимальное абсолютное значение в моменты времени $\tau=\pi / 2+\pi n$, $n=1,2,3 \ldots$. Точка экстремального значения функции мгновенной скорости жидкости (11) в интервале времени $\pi / 2+\pi n \leq \tau \leq \pi / 2+\pi / 8+\pi n$ находится на поверхности пластины.

В первом приближении интеграл (10)

$$
\begin{aligned}
\tilde{\delta}(\tau)= & M_{a} \int_{0}^{1} \exp (-x) \cos (\tau-x) d x=\frac{a}{2}(\sin (\tau) \\
& +\cos (\tau)-\exp (-1)(\sin (\tau-1)+\cos (\tau-1))) .
\end{aligned}
$$

На рис. 2 изображены временные зависимости безразмерной функции давления $p$ и удвоенной толщины вытеснения $\delta$ в приближении (12). Максимальные значения абсолютной величины давления наблюдаются в моменты времени, близкие к значениям $\tau \approx \pi / 2-\pi / 8+\pi n$. Экстремальные значения величины давления находятся на краях рассматриваемой пластины в точке с координатами $x=0, y= \pm(l / 2-0.01)$. Аналогичная закономерность имелась на полюсах колеблющегося цилиндра в

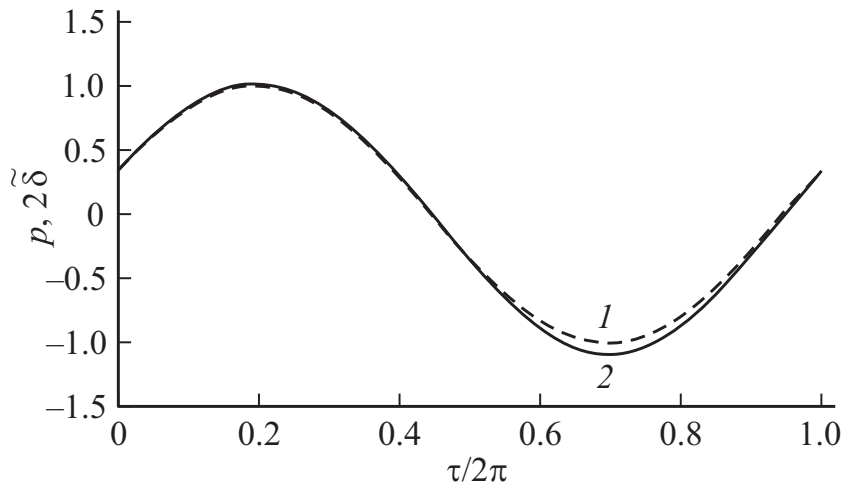

Рис. 2. Сравнение временных зависимостей: 1 - удвоенной толщины вытеснения $\tilde{\delta}(11), 2-$ величины безразмерного давления на крае поверхности пластины при значении $\max |p|=\sqrt{2}$. Зависимость $\tilde{\delta}(\tau)$ смещена по оси абсцисс вправо на величину $3 \pi / 376$ относительно ее фактического значения.

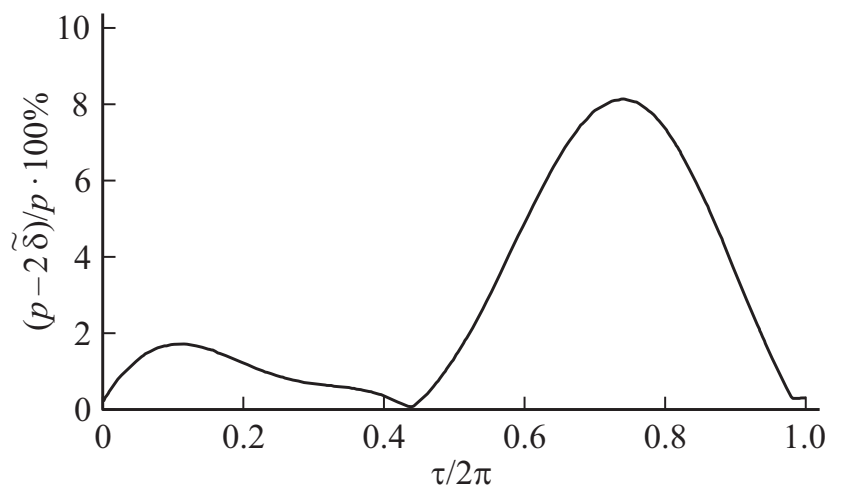

Рис. 3. Зависимость ошибки аппроксимации давления по формуле (12) от времени $\tau$ в процентах.

работе [14]. Максимальное значение абсолютной величины давления составляет $\sqrt{2} \eta \omega$.

В моменты времени $\tau \approx \pi / 2-\pi / 7.52+\pi n$ реализуется максимальная толщина вытеснения $\tilde{\delta}$. Максимум толщины вытеснения возникает раньше максимума величины давления. Различие по времени возникновения максимумов обсуждаемых величин составляет $\Delta \tau \approx 3 \pi / 376$. При значениях параметров $l=1$, $M_{a}=0.5$ на краях поверхности пластины давление выражается по эмпирической приближенной формуле

$$
P \approx \sqrt{2} \eta \omega \cdot 2 \tilde{\delta}(\tau-3 \pi / 376) .
$$

Из рис. 3 видно, что величина ошибки этой аппроксимации не превышает 9\%. Достаточно большая величина ошибки свидетельствует о том, что при указанных значениях параметров $l, M_{a}$ нелинейность задачи уже проявляется. Различие между высотой пиков в интервалах времени $2 \pi n \leq \tau<\pi+2 \pi n$ и $\pi+2 \pi n \leq \tau<2 \pi+2 \pi n$ составляет $7 \%$.

Эффективный поперечный размер такой пластины равен удвоенной толщине вытеснения $2 \tilde{\delta}(\tau)$. Множитель 2 
соответствует наличию у пластины двух граней, соприкасающихся с вязкой жидкостью. Наличие у пластины эффективной толщины $2 \tilde{\delta}(\tau)$ приводит к возникновению потоков вытеснения.

\section{Влияние аналога числа Маха на поля давления и скорости жидкости в установившемся режиме колебаний}

На рис. 4 изображены зависимости безразмерного давления $p$ от времени $\tau$ на краю пластины в точке с безразмерными координатами $x=0, y=l / 2$. Полученные зависимости (кривые 2 и 3) иллюстрируют генерацию высших гармоник за счет нелинейности при увеличении параметра $M_{a}>1$. Модуль величины минимума давления в разы больше, чем модуль величины его максимума. С ростом величины параметра $M_{a}>1$ модули максимума и минимума давления увеличиваются. На рис. 4. это выражено ростом величины $\max p$. Пики зависимостей

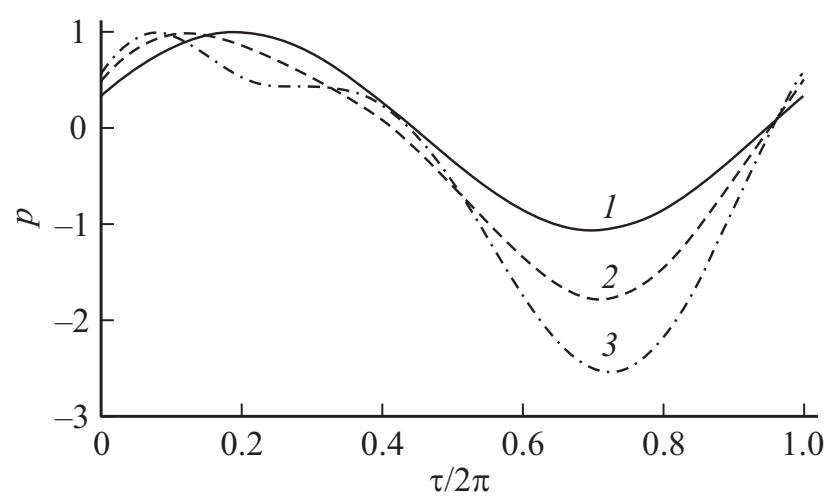

Рис. 4. Значения величин нормированного безразмерного давления $p$, полученных за интервал времени от $3 T+T / 2$ до $4 T$, при разных значениях безразмерного параметра $a$ на крае пластины. Параметр $l=1$. Безразмерные координаты $x=0, y=l / 2-\Delta h .1-M_{a}=0.5, \max |p|=\sqrt{2}, 2-M_{a}=5$, $\max |p|=7.5 \sqrt{2}, 3-M_{a}=10, \max |p|=12 \sqrt{2}$.

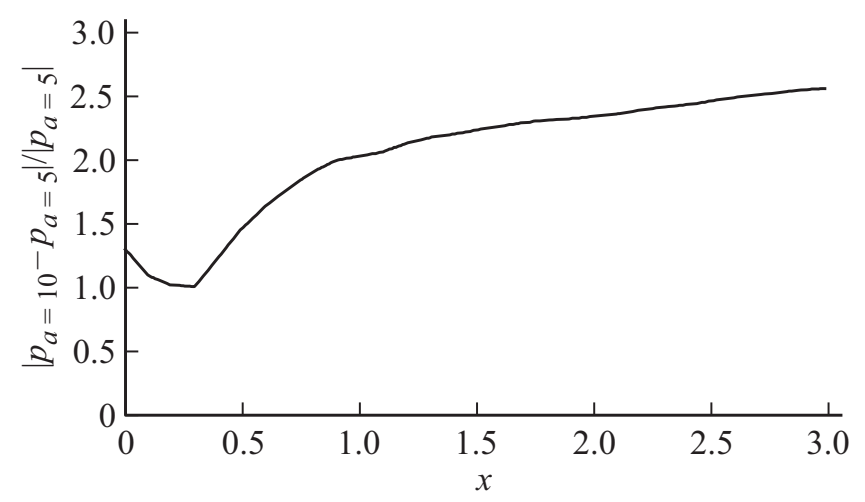

Рис. 5. Различие абсолютных величин безразмерного давления $p$ при значениях безразмерного параметра $M_{a}=5$ и $10 \mathrm{c}$ ростом расстояния $x$ от колеблющейся поверхности. Значение координаты $y=l / 2-\Delta h$. Значение параметра $l=1$.

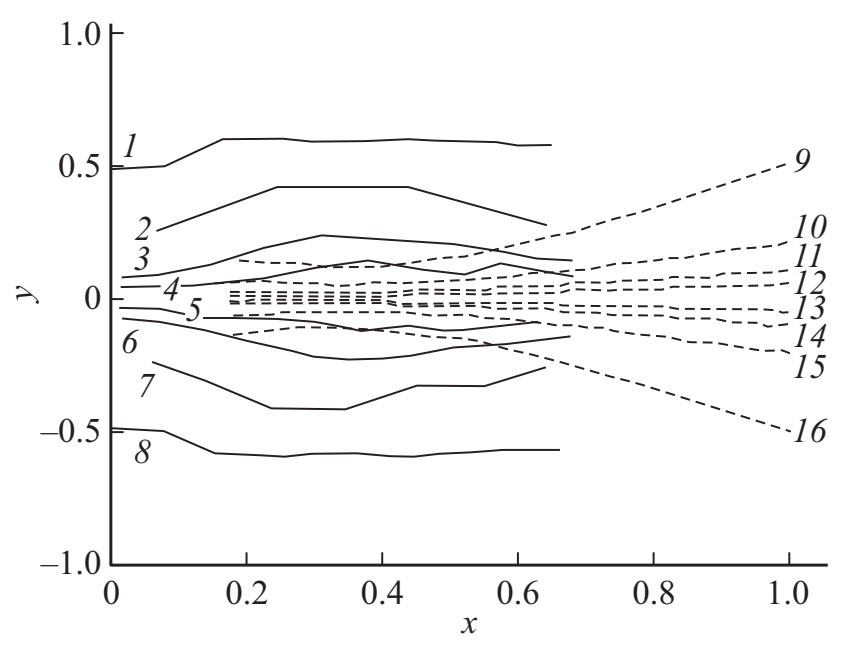

Рис. 6. Траектории перемещения точек нулевого и экстремального значений функции скорости жидкости в нелинейной вязкой волне. Параметр $l=1$. Кривые $1-8$ соответствуют траекториям перемещения точки экстремума скорости жидкости в вязкой волне. Кривые 9-16 соответствуют траекториям перемещения точки нуля скорости жидкости в вязкой волне. Кривые $1-4$ и 9-12 соответствуют интервалу времени $\tau$ от $2 \pi n$ до $\pi+2 \pi n$. Кривые $5-8$ и $13-16$ соответствуют интервалу времени $\tau$ от $\pi+2 \pi n$ до $2 \pi+2 \pi n$. $1,8,9,16-$ параметр $M_{a}=5 ; 2,7,10,15-M_{a}=2 ; 3,6,11,14-M_{a}=1$; $4,5,12,13-M_{a}=0.5$.

сдвигаются по временной шкале левее в интервале времени $2 \pi n \leq \tau<\pi+2 \pi n$ и правее в интервале времени $\pi+2 \pi n \leq \tau<2 \pi+2 \pi n$. Приближение (11) не дает правильного результата. Для корректного вычисления величины $\tilde{\delta}$ требуется подставить результаты численного решения системы (1)-(3) в интеграл (10).

На рис. 5 приведено различие минимальных значений величин давления $p$, полученных при разных значениях параметра $M_{a}$, в окрестности указанного края поверхности пластины с ростом значения безразмерной координаты $x$. При значении $x=1$ минимумы величины давления равны. На расстояниях $x<1$ различие убывает. С ростом расстояния от колеблющейся поверхности при значении $x>1$ различие возрастает.

В работе [13] было замечено искривление траекторий перемещения точек нулевого и экстремального значений функции скорости жидкости при распространении вязкой волны, генерируемой цилиндрами конечных радиусов. Эти траектории не совпадали одна с другой и зависели от параметров задачи. В настоящей работе также замечено искривление этих траекторий. На рис. 6 приведены полученные по результатам численного моделирования траектории перемещения точек нулевого и экстремального значений функции скорости жидкости в нелинейной вязкой волне. Чем больше параметр $M_{a}$, тем больше кривизна траекторий и тем дальше они отходят от центра пластины. Кривизна траекторий свидетельствует [13] о невозможности применения „идеализации поперечной вязкой волны“ при ее распространении от 

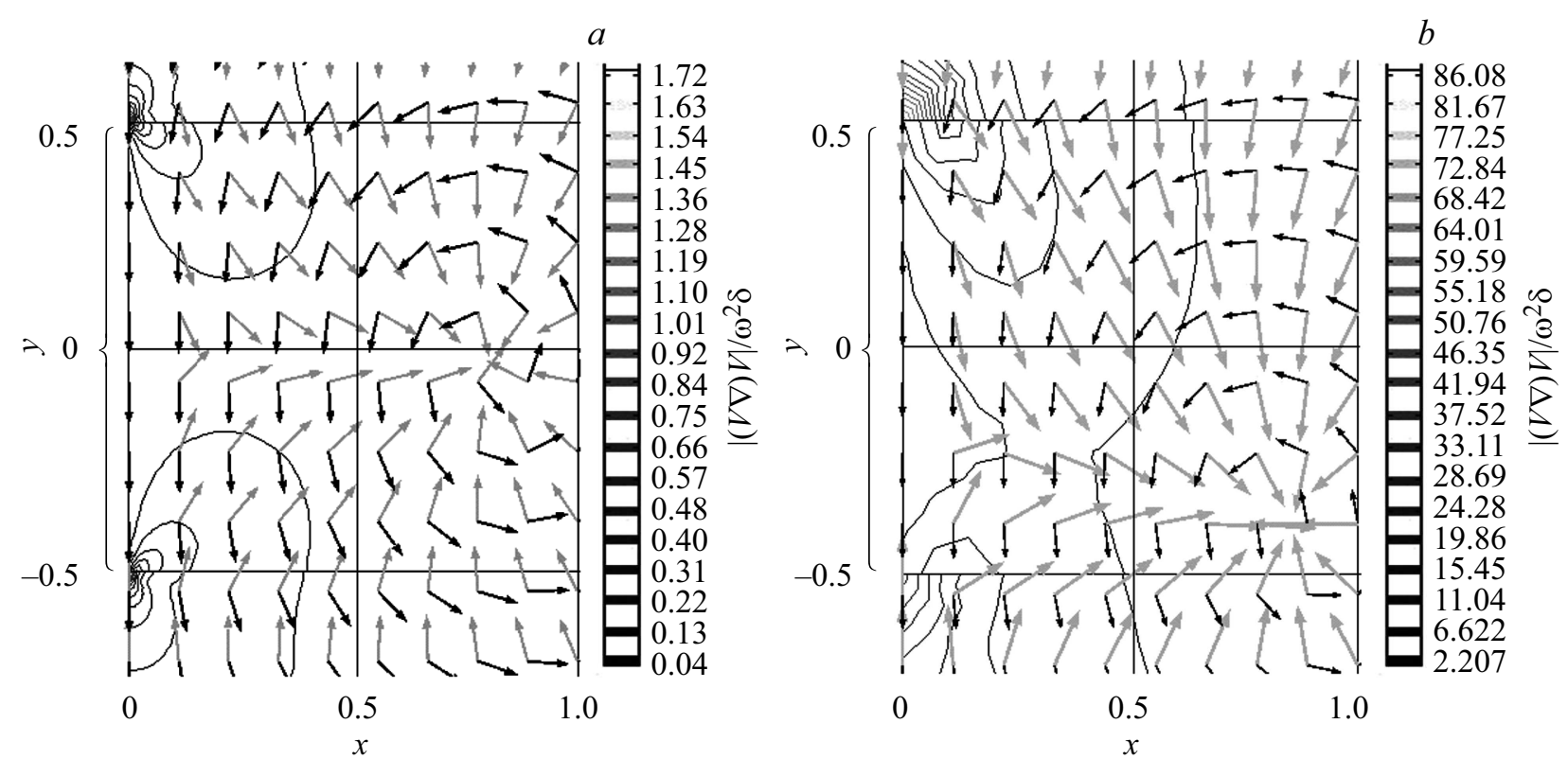

Рис. 7. Конвективное ускорение. Светлые стрелки - направление мгновенного вектора конвективного ускорения. Изолинии - его безразмерная абсолютная величина. Черные стрелки - направление вектора скорости жидкости. Колеблющаяся пластина помечена фигурной скобкой. Значение параметра $l=1$. Момент времени $\tau=3 \pi / 2$. $a-$ значение параметра $M_{a}=0.5$, $b-M_{a}=5.0$.

колеблющейся пластины бесконечно малой толщины, ограниченной в направлении колебаний.

Полученные по результатам численного моделирования безразмерные скорости перемещения точек нулевого и экстремального значений функции скорости жидкости близки к единице.

\section{Роль конвективного ускорения в проявлении нелинейных эффектов}

Выясним, какая сила приводит к возникновению наблюдаемых мгновенных вихревых движений в вязкой жидкости. Роль первого и четвертого слагаемого уравнения (1) ясна из линейной задачи [1]. Происходит генерация вязких волн. Влиянию градиента давления посвящены два раздела настоящей работы. Градиент давления генерирует потоки вытеснения. Потоки вытеснения замыкают линии тока жидкости вдали от поверхности пластины. Однако остается неясным, почему вблизи поверхности пластины существует мгновенный вихрь, центром которого практически во всем интервале времени от $(n-1) T / 2$ до $n T / 2$ является точка, а не линия.

Вдоль траекторий 9-16 рис. 6 перемещаются окрестности центров мгновенных вихревых движений.

На рис. 7 показано направление и безразмерная величина конвективного ускорения $(\mathbf{V} \cdot \nabla) \mathbf{V} /\left(\omega^{2} \delta\right)$ при значениях аналога числа Маха 0.5 и 5 . Темными стрелками показано направление вектора скорости жидкости, светлыми стрелками - направление вектора конвективного ускорения. Сплошные линии - это изолинии величины конвективного ускорения. Величина конвективного ускорения максимальна в окрестности краев пластины. На переднем крае по ходу движения пластины величина конвективного ускорения меньше, чем на заднем по ходу движения крае. На краях пластины конвективное ускорение направлено к ее центру. Вблизи поверхности пластины векторы конвективного ускорения и скорости жидкости практически параллельны.

В окрестности центра мгновенного вихревого движения вектор конвективного ускорения практически перпендикулярен вектору скорости жидкости. Вектор конвективного ускорения направлен к его центру. Конвективное ускорение играет роль центростремительного ускорения жидкости.

Отметим наличие переходной области - области, в которой вектор конвективного ускорения не параллелен и не перпендикулярен вектору скорости жидкости. Для этого снова обратимся к рис. 6. Линии 9-16 на рис. 6 начинаются с координаты $x=0.2$, потому что на меньших расстояниях центр вихря не выражен точкой. Указанное расстояние является размером обсуждаемой окрестности поверхности пластины, в которой конвективное ускорение не играет роли центростремительного ускорения. „Центром“ мг мновенного вихря в этой окрестности является линия. Эта линия - практически прямая, параллельная поверхности пластины. На этой линии скорость жидкости практически обращается в нуль. Эта линия перемещается в пространстве со скоростью распространения вязкой волны. Длина ее по 
мере перемещения сокращается и обращается в нуль на расстоянии $x=0.2$.

Оценим величины слагаемых в (1) по результатам численного счета. Максимум абсолютной величины конвективного ускорения составил $\max \left(|(\mathbf{V} \cdot \nabla) \mathbf{V}| /\left(\omega^{2} \delta\right)\right) \approx 1.8$ при значениях параметров $M_{a}=0.5, l=1$. Сравним его с максимальной величиной градиента давления $\max (\delta|\nabla P| / \omega \eta) \approx 295$ при тех же значениях параметров $M_{a}, l$. Различие составляет два порядка и больше. При значениях параметра $M_{a}>1$ величины максимумов градиента давления и конвективного ускорения отличаются в меньшей степени. Например, при значении параметра $M_{a}=5$ получены следующие значения: $\max \left(|(\mathbf{V} \cdot \nabla) \mathbf{V}| /\left(\omega^{2} \delta\right)\right) \approx$ $\approx 87, \max (\delta|\nabla P| / \omega \eta) \approx 200$. При значениях параметра $M_{a}<1$ поля вязких волн в большей степени искажаются потоками вытеснения.

\section{Заключение}

Вязкие волны, генерируемые колеблющимися телами - источниками конечных размеров вдоль направления колебаний, являются нелинейными.

Результаты настоящей работы свидетельствуют о появлении в вязкой жидкости меняющейся со временем толщины вытеснения у колеблющейся в своей плоскости пластины бесконечно малого размера поперек направления колебаний. При колебаниях в вязкой жидкости пластины бесконечно малой толщины и конечного размера вдоль направления колебаний ее толщина эффективно увеличивается. Образуются область вытеснения и поток вытеснения.

Нелинейные искажения совокупного поля вязких волн и потоков вытеснения проявляются тем сильнее, чем больше величина относительной амплитуды колебаний пластины - параметр $M_{a}$, и чем меньше ее относительная безразмерная длина вдоль направления колебаний параметр $l$. Параметр $M_{a}$ является аналогом числа Маха в смысле отношения амплитуды скорости частицы жидкости к скорости распространения вязковолнового процесса.

Траектории перемещения точек экстремального и нулевого значений скорости жидкости в поле нелинейной вязкой волны с ростом числа Маха становятся криволинейными. Траектория перемещения точки нулевого значения скорости жидкости является центром мгновенного вихря.

Конвективное ускорение играет роль центростремительного ускорения жидкости, направленного к центру мгновенного вихря.

\section{Конфликт интересов}

Авторы заявляют, что у них нет конфликта интересов.

\section{Список литературы}

[1] Ландау Л.Д., Лифиии, Е.M. Теоретическая физика: учебное пособие в 10 т. T. VI: Гидродинамика. 3-е изд., перераб., М.: Наука, 1986. 736 с.

[2] Павловский А.С., Пугачёв С.И., Семёнова Н.Г. // Учен. зап. физ. фак. Моск. ун-та, 2014. № 5. С. 1-7.

[3] Пугачёв С.И., Рытов Е.Ю., Семёнова Н.Г., Павловский А.С. // Морские интеллектуальные технологии. 2014. T. 2. № 2 (24). C. 65-70.

[4] Красавина М.А., Пугачёв С.И., Семёнова Н.Г. Ультразвуковая металлизация электрофизической керамики. СПб.: Изд-во Политех. ун-та, 2003. 184 с.

[5] Станкевич В.Ю., Малышкина О.В., Павловский А.С., Легуша Ф.Ф., Пугачев С.И., Краснов А.В., Александрова П.В., Семёнова Н.Г. Способ изготовления составного электроакустического преобразователя. RU2554591. МПК H04R31/00. 2013148513/28(075453).

[6] Sapozhnikov O.A., Bailey M.R. // J. Acoust. Soc. Am. 2013. Vol. 133. N 2. P. 661-676.

[7] Курзин В.Б. // Прикладная механика и техническая физика. 2011. Т. 52. № 3. С. 153-158.

[8] Павлов В.А., Павловский А.С., Семёнова Н.Г. // ЖТФ. 2016. Т. 86. Вып. 7. С. 31-37.

[9] Павловский А.С., Семёнова Н.Г. // Письма в ЖТФ. 2014. Т. 40. Вып. 8. С. 14-22.

[10] Лойиянский Л.Г. Ламинарный пограничный слой. М.: Физматлит, 1962. 479 с.

[11] Ван-Дайк М. Методы возмущений в механике жидкостей. М.: Мир, 1967. С. 310.

[12] Бетяев С.К. // УФН. 1995. Т. 165. С. 299-330.

[13] Калиткин Н.Н. Численные методы. СПб. 2014.

[14] Frei $W$. How to Identify and Resolve Singularities in the Model when Meshing — https://www.comsol.com/blogs/howidentify-resolve-singularities-model-meshing, 2013. 